\title{
CORRECTION
}

\section{Correction: Clinical epigenomics: genome-wide DNA methylation analysis for the diagnosis of Mendelian disorders}

Bekim Sadikovic (D), Michael A. Levy, Jennifer Kerkhof, Erfan Aref-Eshghi, Laila Schenkel, Alan Stuart, Haley McConkey, Peter Henneman, Andrea Venema, Charles E. Schwartz, Roger E. Stevenson, Steven A. Skinner, Barbara R. DuPont, Robin S. Fletcher, Tugce B. Balci, Victoria Mok Siu, Jorge L. Granadillo, Jennefer Masters, Mike Kadour, Michael J. Friez, Mieke M. van Haelst, Marcel M. A. M. Mannens, Raymond J. Louie, Jennifer A. Lee, Matthew L. Tedder and Marielle Alders

Genetics in Medicine (2021) 23:2228; https://doi.org/10.1038/s41436-021-01130-z

Correction to: Genetics in Medicine (2021); https://doi.org/10.1038/ s41436-020-01096-4, published online 05 February 2021

The original version of this article unfortunately contained a mistake. The following correction has therefore been made in the original: The spelling of Michael A. Levy's name was incorrect. The corrected author list is given above. The original article has been corrected. 